\title{
RADICALS IN CATEGORIES
}

\author{
by MICHAEL HOLCOMBE and ROLAND WALKER
}

(Received 18th November 1976)

\section{Introduction}

The study of radicals in general categories has followed several lines of development. The problem of defining radical properties in general categories has been considered by Kurosh and Shul'geifer, see (7). Under mild conditions on their categories they obtain sufficient conditions for the existence of radical functors which are closely related to radical properties. Another approach is by Maranda (5) and Dickson (3) who studied idempotent radical functors and torsion theories in abelian categories. Our aim has been to study radical functors in as general a category as possible. To this end we introduce the concept of an $\boldsymbol{R}$-category. The categories of rings, modules, near-rings, groups and Jordan algebras are all examples of $\boldsymbol{R}$ categories.

In the first part we describe a general procedure for producing radical functors in $\boldsymbol{R}$-categories and show that every radical functor is obtainable by this procedure.

Although our procedure produces all radicals in abelian categories it does not give the common radicals of ring theory etc. To produce these we amend our definition of radical functor so that its domain is a certain subcategory of the $R$-category. In a similar way all such functors are classified and these give rise to the usual radicals in ring theory etc. Carreau (2) and Wiegandt (8) have obtained partial characterisations of radical functors under different conditions.

In the second half of this paper we examine the problem of describing a suitable generalisation of the concept of a special radical from the theory of rings to the theory of categories. This is then applied to the case of the theory of near-rings and gives us some interesting new insights into the radicals of near-rings. All that is needed is enough structure in our categories to enable the first and second isomorphism theorems to hold.

A general reference for terminology and presentation is Mitchell (6).

1.

In this section we introduce the categories in which we propose to define radical functors. We require first two definitions:

Definition 1.1 A category $\mathscr{C}$ is said to have $N$-intersections if, given an y object $X$ of $\mathscr{C}$ and any set $\left\{u_{i}: X_{i} \rightarrow X \mid i \in I\right\}$ of kernels of $\mathscr{C}$, then the intersection $\cap_{i \in I} u_{i}: \cap_{i \in I} X_{i} \rightarrow X$ exists. 
Definition 1.2. A category $\mathscr{C}$ is called $N$-locally small if every object $A$ of $\mathscr{C}$ possesses a representative set for its normal subobjects. [If $\left\{A_{i} \mid i \in I\right\}$ is a representative set for the normal subobjects of $A$ and for each $i \in I, g_{i}: A_{i} \rightarrow A$ then we sometimes refer to $\left\{g_{i} \mid i \in I\right\}$ as a representative set of kernels of $\left.A\right]$.

Now we describe the type of categories in which our theory is developed.

Definition 1.3. A category $\mathscr{C}$ is said to be an $\boldsymbol{R}$-category if

(i) $\mathscr{C}$ has kernels and cokernels,

(ii) $\mathscr{C}$ has $N$-intersections and is $N$-locally small,

(iii) every morphism of $\mathscr{C}$ factors through a cokernel followed by a monomorphism,

(iv) for every cokernel $t: X \rightarrow Y$ and kernel $Z \rightarrow Y$ we have $t\left(t^{-1}(Z)\right)=Z$.

These conditions seem to be fairly easily investigated in any specific category and were chosen because of this.

The following elementary results are recorded without proof. They can be established in more general categories than $\boldsymbol{R}$-categories and this is indicated.

Lemma 1.4. Let $\mathscr{C}$ be an arbitrary category

(i) if $A_{1} \subseteq A_{2} \subseteq A$ are objects of $\mathscr{C}$ and $A_{1}$ is a normal subobject of $A$ then $A_{1}$ is a normal subobject of $A_{2}$.

(ii) if $\mathscr{C}$ has kernels and cokernels and $f: K \rightarrow A$ is a kernel then coker(ker $f)=f$.

(iii) if $\mathscr{C}$ has kernels then $\mathscr{C}$ has inverse images of normal subobjects and these inverse images are normal. If $f: A \rightarrow B$ and $B^{\prime} \rightarrow B$ is the kernel of $B \rightarrow B^{\prime \prime}$, then $f^{-1}\left(B^{\prime}\right) \rightarrow A$ is the kernel of $A \rightarrow B \rightarrow B^{\prime \prime}$.

Lemma 1.5. Let $\mathscr{C}$ be a category with kernels, cokernels and $N$-intersections. If $\left\{u_{i}: X_{i} \rightarrow X \mid i \in I\right\}$ is a set of kernels in $\mathscr{C}$, then $\cap_{i \in I} u_{i}: \cap_{i \in I} X_{i} \rightarrow X$ is a kernel in $\mathscr{C}$.

Corollary 1.6. Let $\mathscr{C}$ be a category with kernels, cokernels and $N$-intersections. If $\left\{B_{i} \rightarrow B \mid i \in I\right\}$ is a set of kernels and $f: A \rightarrow B$ then $f^{-1}\left(\cap_{i \in I} B_{i}\right)$ exists and $f^{-1}\left(\cap_{i \in I} B_{i}\right)=$ $\cap_{i \in I} f^{-1}\left(B_{i}\right)$.

Lemma 1.7. Let $\mathscr{C}$ be a category with kernels and cokernels. Consider the commutative diagram

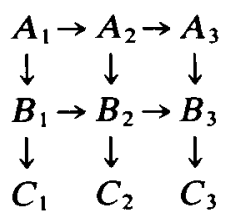

If the initial morphisms of each row and column are kernels and the final morphisms are cokernels of the initial morphisms, then there exist morphisms $C_{1} \rightarrow C_{2}, C_{2} \rightarrow C_{3}$ such that the augmented diagram is commutative and $C_{2} \rightarrow C_{3}=\operatorname{coker}\left(C_{1} \rightarrow C_{2}\right)$.

Corollary 1.8. Let $\mathscr{C}$ be a category with kernels and cokernels, such that every morphism $\alpha: A \rightarrow B$ of $\mathscr{C}$ can be factored into $A \rightarrow I \rightarrow B$ where $A \rightarrow I$ is a cokernel and $I \rightarrow B$ is a monomorphism. Then, in the diagram of Lemma 1.7, $C_{1} \rightarrow C_{2}$ is a monomorphism. 
Lemma 1.9. Let $\mathscr{C}$ be a category with kernels and cokernels, such that every morphism $\alpha: A \rightarrow B$ of $\mathscr{C}$ can be factored into $A \rightarrow I \rightarrow B$ where $A \rightarrow I$ is a cokernel and $I \rightarrow B$ is a monomorphism. Then $\mathscr{C}$ has epimorphic images, $I \rightarrow B$ being the epimorphic image of $\alpha: A \rightarrow B$.

One of the essentials of our theory is a generalisation to $\boldsymbol{R}$-categories of the First Isomorphism Theorem of abelian categories. We require a preliminary result.

Lemma 1.10. Let $\mathscr{C}$ be an $R$-category. Let $t: X \rightarrow Y$ be a cokernel with kernel $Z \rightarrow X$ and let $W \rightarrow X$ be any kernel such that $Z \subseteq W$. Then $t(W) \rightarrow Y$ is a kernel.

Proof. Suppose $X \rightarrow T=\operatorname{Coker}(W \rightarrow X)$. Then $W \rightarrow X=\operatorname{ker}(X \rightarrow T)$; so there exists a morphism $Y \rightarrow T$ such that the diagram

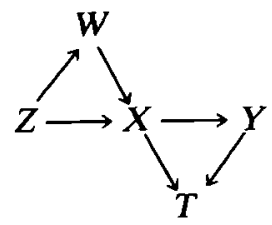

is commutative since $Z \rightarrow X \rightarrow T=Z \rightarrow W \rightarrow X \rightarrow T=0$. Let $K \rightarrow Y=\operatorname{ker}(Y \rightarrow T)$. Now $W \rightarrow X \rightarrow Y \rightarrow T=W \rightarrow X \rightarrow T=0$; hence $W \rightarrow t(W) \rightarrow Y \rightarrow T=0$.

Now $W \rightarrow t(W)$ is a cokernel; thus $t(W) \rightarrow Y \rightarrow T=0$. It follows that a monomorphism $t(W) \rightarrow K$ exists such that $t(W) \rightarrow Y=t(W) \rightarrow K \rightarrow Y$. Hence $t(W) \subseteq K$. Since $t\left(t^{-1}(K)\right) \subseteq K$, we have a commutative diagram

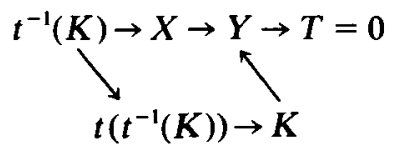

so $t^{-1}(K) \rightarrow X \rightarrow T=0$. But $W \rightarrow X=\operatorname{ker}(X \rightarrow T)$; so there exists a monomorphism $t^{-1}(K) \rightarrow W$ such that $t^{-1}(K) \rightarrow X=t^{-1}(K) \rightarrow W \rightarrow X$. Hence $t^{-1}(K) \subseteq W$ and $t\left(t^{-1}(K)\right) \subseteq t(W)$. Since $\mathscr{C}$ is an $R$-category, $t\left(t^{-1}(K)\right)=K$ we have $K \subseteq t(W)$. Thus we conclude that $K=t(W)$; so $t(W) \rightarrow Y=\operatorname{ker}(Y \rightarrow T)$.

Theorem 1.11 Let $\mathscr{C}$ be an $R$-category. If $A_{1} \rightarrow A$ and $A_{2} \rightarrow A$ are kernels such that $A_{1} \subseteq A_{2}$ then $\left(A / A_{1}\right) /\left(A_{2} / A_{1}\right)$ is isomorphic to $A / A_{2}$.

Proof. $A_{1} \rightarrow A_{2}$ is a kernel by Lemma 1.4. Hence we have a commutative diagram

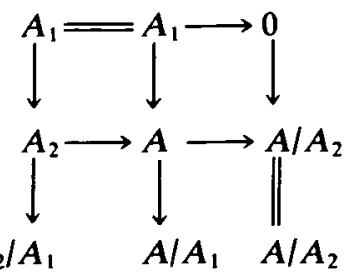


satisfying the hypotheses of Lemma 1.7. From this lemma and Corollary 1.8 we conclude that there exist morphisms $A_{2} / A_{1} \rightarrow A / A_{1}, A / A_{1} \rightarrow A / A_{2}$ such that

(i) the diagram is commutative,

(ii) $A_{2} / A_{1} \rightarrow A / A_{1}$ is a monomorphism, and (iii) $A / A_{1} \rightarrow A / A_{2}=\operatorname{coker}\left(A_{2} / A_{1} \rightarrow A / A_{1}\right)$.

It only remains to show that $A_{2} / A_{1} \rightarrow A / A_{1}=\operatorname{ker}\left(A / A_{1} \rightarrow A / A_{2}\right)$. Let $t: A \rightarrow A / A_{1}$ be the cokernel of $A_{1} \rightarrow A$. Since $A_{1} \subseteq A_{2}$, it follows by Lemma 1.9 that $t\left(A_{2}\right) \rightarrow A / A_{1}$ is a kernel. Now $A_{2} \rightarrow A / A_{1}=A_{2} \rightarrow A_{2} / A_{1} \rightarrow A / A_{1}$, where $A_{2} \rightarrow A_{2} / A_{1}$ is a cokernel and $A_{2} / A_{1} \rightarrow A / A_{1}$ is a monomorphism; so by Lemma $1.7, A_{2} / A_{1} \rightarrow A / A_{1}$ is the image of $A_{2} \rightarrow A / A_{1}$, i.e. $A_{2} / A_{1}=t\left(A_{2}\right)$.

Since $A_{2} / A_{1} \rightarrow A / A_{1}$ is a kernel with cokernel $A / A_{1} \rightarrow A / A_{2}$,

$$
A_{2} / A_{1} \rightarrow A / A_{1}=\operatorname{ker}\left(A / A_{1} \rightarrow A / A_{2}\right)
$$

the result follows.

\section{The classification of $\boldsymbol{r}$-functors}

Definition 2.1. Let $\mathscr{C}$ be an $\boldsymbol{R}$-category. A convariant functor $r: \mathscr{C} \rightarrow \mathscr{C}$ is called an -functor if

(i) $r$ is a subfunctor of the identity functor on $\mathscr{C}$,

(ii) for all $C \in \mathscr{C}, r(C)$ is a normal subobject of $\mathscr{C}$,

(iii) for all $C \in \mathscr{C}, r(C / r(C))=0$.

This is a natural generalisation of the concept of a radical functor in an Abelian category.

Definition 2.2. Let $\mathscr{M}$ be a non-empty class of objects of an $R$-category $\mathscr{C}$. For each $A \in \mathscr{C}$ we define the subobject

$$
\operatorname{rad}_{\mathcal{M}} A=\cap\left\{\operatorname{ker} \alpha \mid \alpha \in[A: M]_{\mathscr{\zeta}}, \quad M \in \mathcal{M}\right\}
$$

Note that we may represent the class of morphisms which arise as kernels of morphisms in $\left\{[A: M]_{\mathscr{B}} \mid M \in M\right\}$ as a set, since $\mathscr{C}$ is $N$-locally small. In many situations we denote this set by $\left\{g_{i}: A_{i} \rightarrow A \mid i \in I\right\}$ and then $\operatorname{rad}_{\mathcal{M}} A \stackrel{\cap_{B_{i}}}{\longrightarrow} A$ is a kernel by Lemma 1.1.

Theorem 2.3. Suppose that $\mathscr{C}$ is an $R$-category and $\mathcal{M}$ is a non-empty class of objects of $C$. If $A \in C$ and $B$ is a normal subobject of $A$ such that $B \subseteq \operatorname{rad}_{\cdot k} A$, then

$$
\operatorname{rad}_{\mathcal{H}}(A / B)=\left(\operatorname{rad}_{. \mu} A\right) / B
$$

Proof. From the results in $\S 1, B \rightarrow \operatorname{rad}_{\mathcal{M}} A$ is a kernel. Consider the set $\left\{g_{i}: A_{i} \rightarrow\right.$ $A \mid i \in I\}$ of kernels of morphisms in $\left\{[A: M]_{\mathscr{C}}, M \in M\right\}$; then $B \rightarrow A=B \rightarrow A_{i} \stackrel{B_{i}}{\longrightarrow} A$ for all $i \in I$, where $B \rightarrow A_{i}=B \rightarrow \operatorname{rad}_{\mathcal{H}} A \rightarrow A_{i}$, and thus $B \rightarrow A_{i}$ is a kernel for all $i \in I$ by Lemma 1.4 .

We show first that for every morphism $u$ from $A / B$ to an object $M$ in $\mathcal{M}$ there is 
an index $i$ in $I$ such that $\operatorname{ker} u=\left(A_{i} / B \rightarrow A / B\right)$. To do this, suppose $\operatorname{ker} u=X \rightarrow A / B$. If $t: A \rightarrow A / B$ is the cokernel of $B \rightarrow A$, then $A \rightarrow A / B \rightarrow M$ has kernel $A_{i} \rightarrow A$ for some $i \in I$ and $t^{-1}(X) \rightarrow A \rightarrow A / B \rightarrow M=0$ implying $t^{-1}(X) \subseteq A_{i}$. Thus $X=$ $t\left(t^{-1}(X)\right) \subseteq t\left(A_{i}\right)$. From the proof of the isomorphism theorem, $t\left(A_{i}\right)=A_{i} / B$ and hence $X \subseteq A_{i} / B$. However $A_{i} \rightarrow A \rightarrow A / B \rightarrow M=0$ so $A_{i} \rightarrow t\left(A_{i}\right) \rightarrow A / B \rightarrow M=0$. Now $A_{i} \rightarrow$ $t\left(A_{i}\right)$ is an epimorphism with $t\left(A_{i}\right)=A_{i} / B$ and therefore $A_{i} / B \rightarrow A / B \rightarrow M=0$ whence $X=A_{i} / B$.

Next we show that for every index $i$ in $I$ there is a morphism from $A / B$ to some object $M$ in $\mathcal{M}$ with kernel $\left(A_{i} / B \rightarrow A / B\right)$. Suppose $g_{i}: A_{i} \rightarrow A$ is the kernel of $(A \rightarrow M)$. We have $A \rightarrow M=A \rightarrow A / A_{i} \rightarrow M$ where $A \rightarrow A / A_{i}$ is the cokernel of $A_{i} \rightarrow A$ and $A / A_{i} \rightarrow M$ is a monomorphism. Consider the commutative diagram

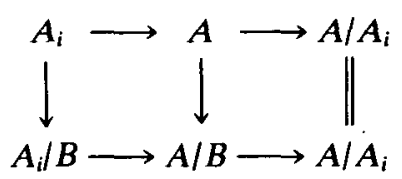

where the vertical morphisms are cokernels, $A_{i} \rightarrow A, A_{i} / B \rightarrow A / B$ are kernels and $A \rightarrow A / A_{i}, A / B \rightarrow A / A_{i}$ their cokernels. Define $A / B \rightarrow M=A / B \rightarrow A / A_{i} \rightarrow M$. Then $\operatorname{ker}(A / B \rightarrow M)=\operatorname{ker}\left(A / B \rightarrow A / A_{i} \rightarrow M\right)=\operatorname{ker}\left(A / B \rightarrow A / A_{i}\right) \quad$ since $\quad A / A_{i} \rightarrow M \quad$ is a monomorphism. Therefore $\operatorname{ker}(A / B \rightarrow M)=A_{i} / B \rightarrow A / B$.

This shows that $\operatorname{rad}_{\mu} A / B=\cap\left(A_{i} / B\right)$.

We finally show that $\cap\left(A_{i} / B\right)=\left(\cap A_{i}\right) / B=\left(\operatorname{rad}_{\mu} A\right) / B$. Let $t: A \rightarrow A / B$ be as before. Then $t^{-1}\left(\cap\left(A_{i} / B\right)\right)=\cap\left(t^{-1}\left(A_{i} / B\right)\right)$. The proof is completed by showing that $t^{-1}\left(A_{i} / B\right)=A_{i}$, for then $\operatorname{rad}_{\mathcal{M}}(A / B)=t\left(t^{-1}\left(\cap\left(A_{i} / B\right)\right)\right)=t\left(\cap A_{i}\right)=t\left(\operatorname{rad}_{\mathcal{M}} A\right)=$ $\left(\operatorname{rad}_{\mathcal{M}} A\right) / B$. Consider the commutative diagram:

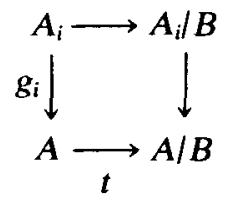

If $Y \rightarrow A, Y \rightarrow A_{i} / B$ are morphisms such that $Y \rightarrow A \rightarrow A / B=Y \rightarrow A_{i} / B \rightarrow A / B$ then $Y \rightarrow A \rightarrow A / B \rightarrow A / A_{i}=Y \rightarrow A_{i} / B \rightarrow A / B \rightarrow A / A_{i}=0$. Thus $Y \rightarrow A \rightarrow A / A_{i}=0$, and so there is a morphism $Y \rightarrow A_{i}$ such that $Y \rightarrow A=Y \rightarrow A_{i} \rightarrow A$. Now $Y \rightarrow A_{i} \rightarrow A_{i} / B \rightarrow$ $A / B=Y \rightarrow A_{i} \rightarrow A \rightarrow A / B=Y \rightarrow A \rightarrow A / B=Y \rightarrow A_{i} / B \rightarrow A / B$. However $A_{i} / B \rightarrow A / B$ is a monomorphism, so $Y \rightarrow A_{i} / B=Y \rightarrow A_{i} \rightarrow A_{i} / B$. This proves that the diagram is a pullback and so $t^{-1}\left(A_{i} / B\right)=A_{i}$.

Corollary 2.4. Let $\mathcal{M}$ be any non-empty class of objects in an $\boldsymbol{R}$-category $\mathscr{C}$. Then $\operatorname{rad}_{\mu}\left(A / \operatorname{rad}_{\mu} A\right)=0$, for all $A \in \mathscr{C}$.

Let $A$ and $B$ be objects of $\mathscr{C}, f \in[A, B]_{\mathscr{C}}$; we propose to define a morphism $\operatorname{rad}_{\mu} f: \operatorname{rad}_{\mathcal{M}} A \rightarrow \operatorname{rad}_{\mathcal{M}} B$. To do this let $\left\{h_{j}: B_{j} \rightarrow B \mid j \in J\right\}$ be a representative set of kernels of morphisms $B \rightarrow M, M \in M$. Given a kernel $h_{j}: B_{j} \rightarrow B$ of a morphism $B \rightarrow M$ then there exists $i \in I$ such that $g_{i}: A_{i} \rightarrow A$ is the kernel of $A \stackrel{\prime}{\rightarrow} B \rightarrow M$. The following diagram is commutative: 


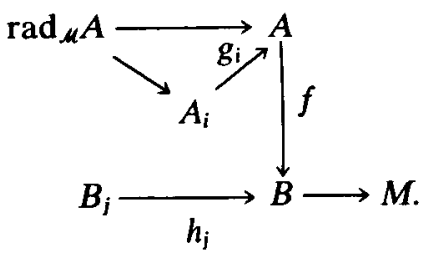

Since $A_{i} \rightarrow A \rightarrow B \rightarrow M=0$, there is a morphism $A_{i} \rightarrow B_{j}$ such that $A_{i} \rightarrow A \rightarrow B=A_{i} \rightarrow$ $B_{j} \rightarrow B$. Therefore there is a morphism $t_{j}: \operatorname{rad}_{\mathcal{M}} A \rightarrow B_{j}$ such that $\operatorname{rad}_{\mathcal{M}} A \rightarrow B_{j}=\operatorname{rad}_{\mathcal{M}} A \rightarrow$ $A_{i} \rightarrow B_{j}$. This is true for each $j \in J$ and so there is a morphism $t: \operatorname{rad}_{\mathcal{M}} A \rightarrow \operatorname{rad}_{\mathcal{H}} B$ such that

$$
\operatorname{rad}_{\mu} A \rightarrow B_{j}=\operatorname{rad}_{\mu} A \rightarrow \operatorname{rad}_{\mu} B \rightarrow B_{j}, \quad \text { for all } j \in J .
$$

Since $A_{i} \rightarrow A \rightarrow B=A_{i} \rightarrow B_{j} \rightarrow B$ we have

$$
\begin{aligned}
\operatorname{rad}_{\mathcal{M}} A \rightarrow A \rightarrow B & =\operatorname{rad}_{\mathcal{M}} A \rightarrow B_{i} \rightarrow B, \quad \text { for all } j \in J \\
& =\operatorname{rad}_{\mathcal{M}} A \rightarrow \operatorname{rad}_{\mathcal{M}} B \rightarrow B_{i} \rightarrow B, \text { for all } j \in J \\
& =\operatorname{rad}_{\mathcal{M}} A \rightarrow \operatorname{rad}_{\mathcal{M}} B \rightarrow B .
\end{aligned}
$$

If we write $\operatorname{rad}_{\mathcal{N}} f=t$, then the diagram

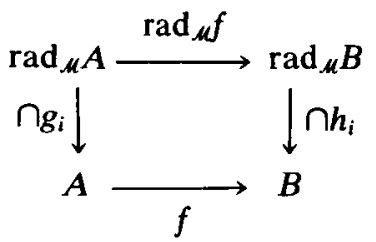

is commutative.

Theorem 2.5. Let $M$ be a non-empty class of objects of an $R$-category $\mathscr{C}$. Then the assignment $\operatorname{rad}_{\mathcal{M}}: A \mapsto \operatorname{rad}_{\mathcal{M}} A$, is an $\bullet$-functor in $\mathscr{C}$.

Proof. By Lemma $1.5 \operatorname{rad}_{\mu} A$ is a normal subobject for each $A$ in $\mathscr{C}$. By Corollary 2.4 we see that $\operatorname{rad}_{\mathcal{M}}\left(A / \operatorname{rad}_{\mu} A\right)=0$ for each $A$ in $\mathscr{C}$. If $f=1_{A}$ in the diagram $(*)$ then

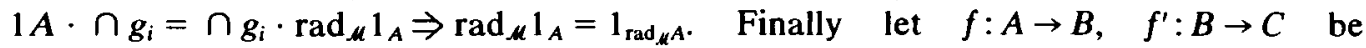
morphisms in $\mathscr{C}$. Denote by $\left\{k_{l}: C_{l} \rightarrow C \mid l \in L\right\}$ a representative set of kernels of morphisms of $C$ into objects $M$ of $\mathcal{M}$. Then $\operatorname{rad}_{\mu} C=\cap C_{l}$. If $t^{\prime}=\operatorname{rad}_{\mu} f^{\prime}$ then the following diagram is commutative:

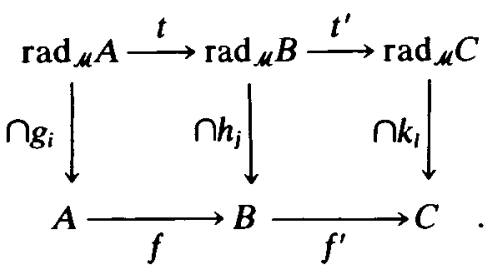

Define $t^{\prime \prime}=\operatorname{rad}_{\mu}\left(f^{\prime} f\right)$, then by construction 


$$
f^{\prime} f \cdot \cap g_{i}=\cap k_{l} \cdot t^{\prime \prime}, \text { thus } n k_{l} \cdot t^{\prime \prime}=n k_{l} \cdot t^{\prime} t
$$

But $\cap k_{l}$ is a monomorphism and so $t^{\prime \prime}=t^{\prime} t$. Therefore $\operatorname{rad}_{\mathcal{M}}: A \rightarrow \operatorname{rad}_{\mathcal{M}} A$ is a covariant functor and is in fact a subfunctor of the identity functor on $\mathscr{C}$, the natural transformation $\eta: \operatorname{rad}_{\mathcal{M}} \rightarrow I_{\mathscr{C}}$ being given by the inclusion $n g_{i}: \operatorname{rad}_{\mathcal{M}} A \rightarrow A, A \in \mathscr{C}$.

Theorem 2.6. Let $\mathscr{C}$ be an $R$-category. A functor $r: \mathscr{C} \rightarrow \mathscr{C}$ is an -functor if and only if $r$ is naturally equivalent to $\operatorname{rad}_{\mathcal{M}}$, for some non-empty class $\mathcal{M}$ of objects of $\mathscr{C}$. If $r$ is an -functor, the class $\mathcal{M}$ can be taken as the class $\{A \in \mathscr{C} \mid r(A)=0\}$.

Proof. We have already established that for each $\mathcal{M} \operatorname{rad}_{\mathcal{A}}$ is an $\ell$-functor. Suppose conversely that $r: \mathscr{C} \rightarrow \mathscr{C}$ is an $t$-functor and let $\mathscr{S}=\{A \in \mathscr{C} \mid r(A)=0\}$. We consider the 2 -functor $\operatorname{rad}_{\varphi}$. Since $r(A / r(A))=0, A / r(A) \in S$ for all $A \in \mathscr{C}$. Now $r(A) \rightarrow A$ is the kernel of $A \rightarrow A / r(A)$ and so $\operatorname{rad}_{\varphi} A \subseteq r(A)$, thus $\operatorname{rad}_{\varphi} A \rightarrow A=\operatorname{rad}_{\varphi} A \rightarrow$ $r(A) \rightarrow A(*)$. Since $\operatorname{rad}_{\varphi} A \rightarrow A$ is a monomorphism, $\operatorname{rad}_{\varphi A} \rightarrow r(A)$ is a monomorphism. Now consider a kernel $A_{i} \rightarrow A$ of a morphism $A \rightarrow S$ where $S \in \mathscr{S}$. Since $r(S)=0$ and $r$ is a subfunctor of the identity functor we have the commutative diagram

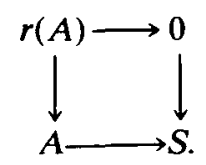

Thus $r(A) \rightarrow A \rightarrow S=0$ and so there exists a morphism $r(A) \rightarrow A_{i}$ such that $r(A) \rightarrow A=$ $r(A) \rightarrow A_{i} \rightarrow A$, and this occurs for each $i \in I$. Consequently there exists a morphism $r(A) \rightarrow \operatorname{rad}_{\varphi}(A)$ such that $r(A) \rightarrow \operatorname{rad}_{\varphi} A \rightarrow A_{i}=r(A) \rightarrow A_{i}$ for every $i \in I$. Now $r(A) \rightarrow A$ is a monomorphism, as also is $r(A) \rightarrow A_{i}$. Thus $r(A) \rightarrow \operatorname{rad}_{s} A$ is a monomorphism. Consequently $r(A) \rightarrow A=r(A) \rightarrow \operatorname{rad}_{\varphi} A \rightarrow A$ and this combined with (*) proves that $r(A) \rightarrow \operatorname{rad}_{\mathscr{S}} A$ is an isomorphism. Hence $r$ and $\operatorname{rad}_{\varphi}$ are naturally equivalent since the correspondence is natural.

This theorem determines all radical functors in an abelian category, and all $\boldsymbol{t}$-functors in an $\boldsymbol{R}$-category. It should be noted that different classes $\mathcal{M}, \mathcal{M}^{\prime}$ of objects of the $\boldsymbol{R}$-category $\mathscr{C}$, could give rise to the same functor $\operatorname{rad}_{\mathcal{M}}=\operatorname{rad}_{\mathcal{M}^{\prime}}$, and in general, when studying an $\mathscr{t}$-functor $r: \mathscr{C} \rightarrow \mathscr{C}$ it is probably more usual to consider it to be represented in the form $\operatorname{rad}_{\mathscr{S}}: \mathscr{C} \rightarrow \mathscr{C}$, where $\mathscr{S}=\{A \in \mathscr{C} \mid r(A)=0\}$, that is the class of ' $r$-semi-simple objects'. Furthermore, for any non-empty class $\mathcal{M}$ of objects of an $\boldsymbol{R}$-category $\mathscr{C}$, we have

$$
\mathcal{M} \subseteq\left\{A \in \mathscr{C} \mid \operatorname{rad}_{\mathcal{M}} A=0\right\},
$$

and so the class of semi-simple objects with respect to the radical functor is the maximum class that can define the functor.

\section{The classification of $R$-functors}

Although the notion of $\boldsymbol{\imath}$-functor defined in $\$ 2$ generalises that of radical functor in an abelian category, it is in some ways unsatisfactory, in that it does not enable us to 
consider those radicals of rings, near-rings, groups, etc. which do not have a representation in an abelian category.

Consider, for example, the category of rings, $\mathscr{R} i$. Let $S$ be a radical property defined on $\mathscr{R} i$, see Divinsky (2), and let $r: \mathscr{R} i \rightarrow \mathscr{R} i$ be the rule which assigns to each ring $R$ its $S$-radical, $S(R)$. Then $r$ is not an $t$-functor, since given a ring-homomorphism $f: R \rightarrow R^{\prime}$, by construction $r(f)=f \mid S(R)$ and $r(f)(S(R))=f(S(R))$, which is an $S$-subring and not necessarily an $S$-ideal of $R^{\prime}$, and so is not necessarily contained in $S\left(R^{\prime}\right)$.

However, we do know that if $f: R \rightarrow R^{\prime}$ is an onto-homomorphism, i.e. a cokernel in $\mathscr{R} i$, then $f(S(R))$ is an $S$-ideal, so $f(S(R)) \subseteq S\left(R^{\prime}\right)$ and $r(f): S(R) \rightarrow S\left(R^{\prime}\right)$. It is then evident that if we let $\mathscr{E}(\mathscr{R} i)$ denote the category whose objects are rings and whose only morphisms are onto-homomorphisms, then $r: \mathscr{E}(\mathscr{R} i) \rightarrow \mathscr{R} i$ defined by $r(R)=$ $S(R)$, and $r(f)=f \mid S(R)$, where $R$ is a ring and $f$ an onto-homomorphism, is a functor, being a subfunctor of the inclusion functor $\mathscr{E}(\mathscr{R} i) \rightarrow \mathscr{R} i$. Of course, we also have the properties that $r(R)$ is an ideal and $r(R / r(R))=0$.

Furthermore, if one considers those well-known radicals which have representations as intersections of ideals, i.e. kernels in $\mathscr{R} i$, one sees that the ideals are the kernels of onto-homomorphisms, i.e. cokernels in $\mathscr{R} i$, into some class of objects.

With this in mind we proceed to develop the theory of $\mathscr{R}$-functors to accommodate this situation.

Theorem 3.1. Let $\mathscr{C}$ be an $\boldsymbol{R}$-category. If $f: A \rightarrow B$ and $g: B \rightarrow C$ are cokernels in $C$ then $g f: A \rightarrow C$ is also a cokernel.

Proof. Let $u: U \rightarrow A, v: V \rightarrow B$ be the kernels of $f, g$ respectively. Then $f=$ coker $u, g=$ coker $v$. From the pullback

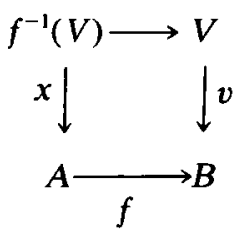

it is clear that $f^{-1}(V) \rightarrow A \rightarrow C=0$, i.e. $(g f) x=0$. We shall show that in fact $x=\operatorname{ker} g f$. So suppose $Y \rightarrow A \rightarrow B \rightarrow C=0$. Then there exists a morphism $Y \rightarrow V$ such that the diagram

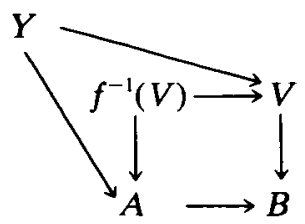

is commutative, and so there exists a morphism $Y \rightarrow f^{-1}(V)$ such that $Y \rightarrow A=Y \rightarrow$ $f^{-1}(V) \rightarrow A$. Hence $x=$ ker $g f$.

We now show that $g f=\operatorname{coker} x$. So suppose $d x=0$ where $d: A \rightarrow D$. We can construct a commutative diagram 


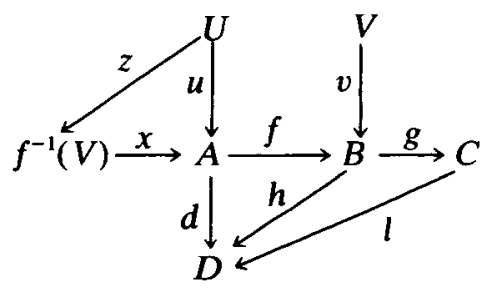

as follows: (1) $f u=0 \Rightarrow g f u=0 \Rightarrow$ there exists a morphism $z: U \rightarrow f^{-1}(V)$ such that $x z=u$,(2) $d x=0 \Rightarrow d x z=0 \Rightarrow d u=0$, so there exists a morphism $h: B \rightarrow D$ such that $d=h f$. Since $f\left(f^{-1}(V)\right)=V$ and $h f x=d x=0$, it follows that $h v=0$, so there exists a morphism $l: C \rightarrow D$ such that $h=\lg$. Finally we see that $d=h f=\lg f$. So $g f=\operatorname{coker} x$.

Definition 3.2. Let $\mathscr{C}$ be an $\boldsymbol{R}$-category. By the cokernel subcategory, $\mathscr{E}(\mathscr{C})$ of $\mathscr{C}$ we mean the subcategory whose objects are the objects of $\mathscr{C}$ and whose only morphisms are the cokernels of $\mathscr{C}$, so $[A: B]_{\mathscr{B}(\mathscr{G})}=\left\{f \in[A: B]_{\mathscr{E}} \mid f\right.$ is a cokernel $\}$. The previous result ensures that $\mathscr{E}(\mathscr{C})$ is a subcategory of $\mathscr{C}$. We now define a new radical functor as follows:

Definition 3.3. Let $\mathscr{C}$ be an $\boldsymbol{R}$-category. A covariant functor $r$ from $\mathscr{E}(\mathscr{C})$ to $\mathscr{C}$ is called an $\mathscr{R}$-functor if

(i) $r$ is a subfunctor of the inclusion functor $j: \mathscr{C}(\mathscr{C}) \rightarrow \mathscr{C}$,

(ii) for all $C \in \mathscr{C}, r(C)$ is a normal subobject of $C$,

(iii) for all $C \in \mathscr{C}, r(C / r(C))=0$.

If $r$ satisfies the further condition

(iv) for all $c \in \mathscr{C}$, if $K$ is a normal subobject of $C$ such that $r(K)=K$, then $K \subseteq r(C)$,

we say that $r$ is complete.

Definition 3.4. Let $\mathcal{M}$ be a non-empty class of objects of an $\boldsymbol{R}$-category $\mathscr{C}$. For each object $A \in \mathscr{C}$, we define the subobject

$$
\operatorname{Rad}_{\mathcal{M}} A=\cap\left\{\operatorname{ker} \alpha \mid \alpha \in[A: M]_{(\mathscr{(})}, \quad M \in \mathcal{M}\right\} .
$$

Corresponding to Theorem 2.3 and its corollary, we have:

Theorem 3.5. Suppose that $\mathscr{C}$ is an $\boldsymbol{R}$-category and $\mathcal{M}$ is a non-empty class of objects of $\mathscr{C}$. If $A \in \mathscr{C}$ and $B$ is a normal subobject of $A$ such that $B \subseteq \operatorname{Rad}_{\mathcal{M}} A$, then $\operatorname{Rad}_{\mathcal{M}}(A / B)=\left(\operatorname{Rad}_{\mathcal{M}} A\right) / B$.

Corollary 3.6. Let $\mathcal{M}$ be any non-empty class of objects in an $\boldsymbol{R}$-category $\mathscr{C}$. Then $\operatorname{Rad}_{\mathcal{M}}\left(A / \operatorname{Rad}_{\mathcal{M}} A\right)=0$, for all $A \in C$.

Theorem 3.7. Let $\mathcal{M}$ be a non-empty class of objects of an $\boldsymbol{R}$-category $\mathscr{C}$. Then the assignment $\operatorname{Rad}_{\mathcal{M}}: A \rightarrow \operatorname{Rad}_{\mathcal{M}} A$ is an $\mathscr{R}$-functor from $\mathscr{E}(\mathscr{C})$ to $\mathscr{C}$.

Proof. The method of proof employed in Theorem 2.5 can again be used. 
However, given $f \in[A: B]_{\mathscr{E}(\mathscr{B})}$, there is a simpler method of constructing $\operatorname{Rad}_{\mu} f$ in this case. If $C \rightarrow A=\operatorname{ker} f$, then $f=\operatorname{coker}(C \rightarrow A)$, so $B=A / C$. Let $\left\{g_{i}: A_{i} \rightarrow A \mid i \in I\right\}$ be a representative set of kernels of cokernels $A \rightarrow M, M \in \mathcal{M}$. Then if $\left\{g_{k}: A_{k} \rightarrow A \mid k \in K \subseteq\right.$ $I\}$ is that subset such that $A_{k} \supseteq C$ if and only if $k \in K$, it is easy to see that the set $\left\{A_{k} / C \rightarrow A / C \mid k \in K\right\}$ is a representative set of kernels of cokernels $A / C \rightarrow M, M \in \mathcal{M}$. Now $\operatorname{Rad}_{\mathcal{M}} A / C=\cap_{k \in K}\left(A_{k} / C\right)=\cap_{k \in K}\left(A_{k}\right) / C$ and there exists a natural morphism $\operatorname{Rad}_{\mu} A \rightarrow \operatorname{Rad}_{\mu} B$ given by

$$
\operatorname{Rad}_{\mathcal{M}} A=\bigcap_{i \in I} A_{i} \rightarrow \bigcap_{k \in K} A_{k} \rightarrow \bigcap_{k \in K} A_{k} / C=\operatorname{Rad}_{\mathcal{M}} A / C=\operatorname{Rad}_{\mathcal{M}} B
$$

such that commutativity of the diagram

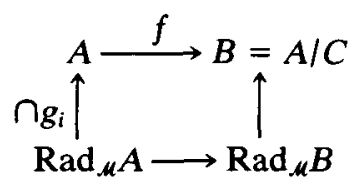

is assured. We take this morphism $\operatorname{Rad}_{\mathcal{M}} A \rightarrow \operatorname{Rad}_{\mathcal{M}} B$ to be $\operatorname{Rad}_{\mathcal{M}} f$.

As before, it is evident from the construction that if $f: A \rightarrow B$ and $g: B \rightarrow C$ are

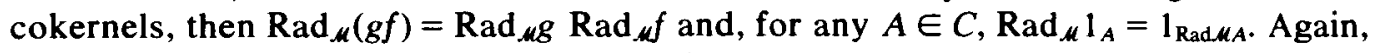
the natural transformation $\eta: \operatorname{Rad}_{\mu} \rightarrow j$ which ensures that $\operatorname{Rad}_{\mathcal{M}}$ is a subfunctor of the inclusion functor is given by the inclusions $\cap g_{i}: \operatorname{Rad}_{\mathcal{M}} A \rightarrow A$, for $A \in \mathscr{C}$.

As one might hope, we can again assert that all $\mathscr{R}$-functors of a given $\boldsymbol{R}$-category $\mathscr{C}$ arise essentially as $\operatorname{Rad}_{\mathcal{M}}$ for some non-empty class $\mathcal{M}$ of objects of $\mathscr{C}$ :

Theorem 3.8. Let $\mathscr{C}$ be an R-category. A functor $r: \mathscr{E}(\mathscr{C}) \rightarrow \mathscr{C}$ is an $\mathscr{R}$-functor if and only if $r$ is naturally equivalent to $\operatorname{Rad}_{\mathcal{M}}$, for some non-empty class $\mathcal{M}$ of objects of $\mathscr{C}$. If $r$ is an $\mathscr{R}$-functor, the class $M$ may be taken as the class $\{A \in \mathscr{C} \mid r(A)=0\}$.

Proof. See Theorem 2.6.

We observe again that different classes $\mathcal{M}, \mathcal{M}^{\prime}$ of an $\boldsymbol{R}$-category $\mathscr{C}$ could give rise to the same $\mathscr{R}$-functor, $r$, but that there is a maximum class $\mathscr{S}$ which can be used to represent $r$ in the form $\operatorname{Rad}_{\mathscr{S}}$, namely the class $\mathscr{S}=\{A \in \mathscr{C} \mid r(A)=0\}$ of " $r$-semi-simple objects". The following definition is a straightforward translation of the definition of a radical property as given by Divinsky (4).

Definition 3.9. Let $\mathscr{C}$ be a category with kernels and cokernels and let $\boldsymbol{S}$ be a property that an object of $\mathscr{C}$ may possess. An object of $\mathscr{C}$ is called an S-object if it possesses property $\boldsymbol{S}$, and is said to be $\boldsymbol{S}$-semi-simple if it contains no non-zero normal $\boldsymbol{S}$-subobjects. $\boldsymbol{S}$ is said to be a radical property if the following conditions hold:

1. if $A$ is an $S$-object and $f: A \rightarrow B$ is a cokernel, then $B$ is an $S$-object,

2. every object $A \in \mathscr{C}$ contains a normal $S$-subobject $S$, which contains every other normal $\boldsymbol{S}$-subobject of $A$,

3. for all $A \in \mathscr{C}, A / S$ is $S$-semi-simple.

$S$ is called the $S$-radical of $A$. 
In studying the relation between radical properties and radical functors, the idea of an idempotent functor has proved important. In the situation in which we find ourselves here, we cannot consider an $\mathscr{R}$-functor to be idempotent as a functor since its domain is not the same as its codomain. However, since the objects of the domain and codomain coincide, we can still talk of an $\mathscr{R}$-functor $r$ as being idempotent if $r(r(A))=r(A)$ for all $A \in C$.

With this weaker notion of idempotence, we can relate $\mathscr{R}$-functors and radical properties in $\boldsymbol{R}$-categories.

Theorem 3.10. Let $C$ be an $R$-category and $r: \mathscr{E}(\mathscr{C}) \rightarrow \mathscr{C}$ be an idempotent complete $\mathscr{R}$-functor. Then the property $S: r(A)=A$ is a radical property and

(i) for all $A \in \mathscr{C}, r(A)$ is the $S$-radical of $A$,

(ii) the class of $S$-semi-simple objects is precisely the class $\{A \in \mathscr{C} \mid r(A)=0\}$ of r-semi-simple objects.

Proof. Let $A$ be an $S$-object and $f: A \rightarrow B$ be a cokernel in $\mathscr{C}$. Since $r(A)=A$ we have a commutative diagram

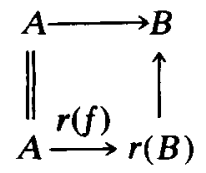

from which it follows that $r(B)=B$, so $B$ is an $S$-object.

By definition of idempotence and completeness, it follows immediately that $r(A)$ is a normal $\boldsymbol{S}$-subobject of $A$, which contains every other normal $S$-subobject of $A$, and since $r(A / r(A))=0, A / r(A)$ is $S$-semi-simple.

Note: If one were to relax the definition of radical property so that the property $S$ should satisfy only two of the three conditions, namely that the class of $\boldsymbol{S}$-objects is closed under cokernels, and that every object $A$ of $\mathscr{C}$ possesses a normal subobject $S$ such that $A / S$ is $S$-semi-simple, then every $\mathscr{R}$-functor $r$ would give rise to a radical property.

As a partial converse of the above result we have:

Theorem 3.11. Let $\mathscr{C}$ be an $\boldsymbol{R}$-category and $\boldsymbol{S}$ be a radical property defined on $\mathscr{C}$. If the property $S$ gives rise to an $\mathscr{R}$-functor $r$ under the assignment $A \rightarrow S(A)$, its $S$-radical, then $r$ is an idempotent complete $\mathscr{R}$-functor and $r=\operatorname{Rad}_{\mathcal{M}}$, where $\mathcal{M}$ is the class of S-semi-simple objects.

Proof. The only thing that needs to be proved is that $r=\operatorname{Rad}_{\mu}$. We first observe that if $r: A \rightarrow S(A)$ is an $\mathscr{R}$-functor, then $S(A)$ is the unique minimal normal subobject of $A$ such that $A / S(A)$ is $S$-semi-simple. Suppose $I \rightarrow A$ is a kernel and $A / I$ is $S$-semi-simple; then from the commutative diagram 


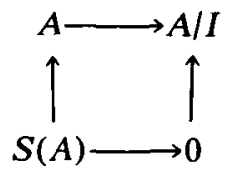

we see that $S(A) \rightarrow A \rightarrow A / I=0$ so $S(A) \subseteq I$.

Let $M \in M$ and suppose that $A \rightarrow M$ is a cokernel with kernel $I \rightarrow A$. Then $M=A / I$ so $I \supseteq S(A)$. Hence $\operatorname{Rad}_{\mathcal{M}} A \supseteq S(A)$. On the other hand, $A / S(A) \in M$ and $S(A) \rightarrow A=$ $\operatorname{ker}(A \rightarrow A / S(A))$; so $S(A) \supseteq \operatorname{Rad}_{\mathcal{M}} A$. Hence $r(A)=\operatorname{Rad}_{\mathcal{M}} A$.

Clearly, if $f: A \rightarrow B$ is a cokernel in $\mathscr{C}, r(f)=\operatorname{Rad}_{\mu} f$.

Note: A sufficient condition for every radical property $\boldsymbol{S}$ of an $\boldsymbol{R}$-category $\mathscr{C}$ to give rise to an $\mathscr{R}$-functor is that $\mathscr{C}$ satisfies the additional condition: If $t: X \rightarrow Y$ is a cokernel and $W \rightarrow X$ is a kernel, then $t(W) \rightarrow Y$ is also a kernel.

\section{Complete radical functors}

Having introduced the notions of idempotence and completeness for $\mathscr{R}$-functors we now turn to the problem of finding conditions on a class $\mathcal{M}$ which will ensure that the corresponding $\mathscr{R}$-functor $\operatorname{Rad}_{\mu}$ enjoys these properties. The motivation for this investigation is as follows: it is known that in the category of rings all radicals are idempotent and complete, but no such general results are known in many other categories. In the category of near-rings it is not known if all the Jacobson type radicals are idempotent. It is thus of interest to study the situation in a general categorical context with the aim of finding tests for idempotence and completeness and possibly discovering whether idempotent and complete radicals are the rule or the exception. It turns out that for completeness the problem is straightforward, but for idempotence it appears to be rather deep.

We assume as before that $\mathscr{C}$ is an $\mathscr{R}$-category, but we also require that $\mathscr{C}$ has arbitrary unions of normal subobjects and that the second isomorphism theorem is valid in $\mathscr{C}$. Thus we require that if $A_{1}, A_{2}$ are normal subobjects of an object $A \in \mathscr{C}$ then

$$
\left(A_{1} \cup A_{2}\right) / A_{1} \approx A_{2} /\left(A_{1} \cap A_{2}\right) \text {. }
$$

Again this can be deduced from the elementary conditions on the category. Let $\mathscr{C}$ be an $\mathscr{R}$-category.

Definition 4.1. A class $\mathscr{Z}$ of objects of $\mathscr{C}$ is said to have condition $(E)$ if for every object $A$ of $\mathscr{X}$ and every non-zero normal subobject $B$ of $A$ there is a non-zero object $X$ in $\mathscr{Z}$ such that $[B: X]_{\mathscr{B}(\mathscr{C})}$ is non-empty. This concept is a straightforward generalisation of the ring theoretic terminology, see, for example Divinsky (1).

Lemma 4.2. Let $r: \mathscr{C}(\mathscr{C}) \rightarrow \mathscr{C}$ be an $\mathscr{R}$-functor with semi-simple class $\mathcal{M}$. Then $r$ is complete if and only if $\mathcal{M}$ has condition $(E)$.

Proof. First we suppose that $r$ is complete. Let $Y \in \mathcal{M}$ and let $X \neq 0$ be such that $X$ is normal in $Y$. If there is no non-zero object $M \in \mathcal{M}$ such that $[X, M]_{\mathscr{B}(\varphi)} \neq \emptyset$, then $r(X)=X$ and so $X \subseteq r(Y)=0$. This is a contradiction. 
Now suppose that $\mathcal{M}$ has condition (E). We first of all note that if $A \rightarrow B$ is a cokernel and $r(A)=A$ then $r(B)=B$, since the diagram

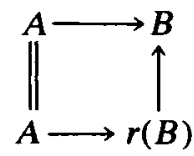

is commutative. Let $K$ be normal in an object $A$ with $r(K)=K$; now $r(K / K \cap r(A))=r(K) /(K \cap r(A))=K /(K \cap r(A))$ and thus $[K /(K \cap r(A)): X]_{(\&(8)}=\emptyset$ for all $X \in M$. However this implies that $\left[(K \cup r(A) / r(A): X]_{g_{(8)}}=\emptyset\right.$ for all $X \in \mathcal{M}$ and yet $(K \cup r(A)) / r(A)$ is normal in $A / r(A)$ which belongs to $\mathcal{M}$. Thus $(K \cup r(A)) / r(A)=0$ and so $K \cup r(A) \subseteq r(A)$ and thus $K \subseteq r(A)$.

Theorem 4.3. If $\mathcal{M}$ is any class of objects of $\mathscr{C}$ satisfying condition $(E)$ then $\operatorname{Rad}_{\mathcal{M}}$ is a complete $\mathscr{R}$-functor.

Proof. Let $\mathcal{N}=\left\{A \in \mathscr{C} \mid \operatorname{Rad}_{\mathcal{M}} A=0\right\}$ be the class of semi-simples with respect to $\operatorname{Rad}_{\mathcal{M}}$. We show that $\mathcal{N}$, too, has condition $(E)$. Suppose that $N \in \mathcal{N}$ and $K \neq 0$ is a normal subobject of $N$. We can find a representative set of cokernels $\left\{f_{i}: N \rightarrow M_{i}\right.$; $\left.M_{i} \in \mathcal{M}, i \in I\right\}$ with kernels $\left\{L_{i} \rightarrow N \mid i \in I\right\}$ such that $\cap_{i \in I} L_{i}=0$. If $f_{i}(K)=0$ for all $i \in I$ then $K \subseteq L_{i}$ for all $i$; so $K \subseteq \cap_{i \in I} L_{i}=0$. Therefore there exists $i_{o} \in I$ such that $f_{i_{o}}(K) \neq 0$. Since $f_{i_{o}}: N \rightarrow M_{i_{o}}$ is a cokernel and $K$ is normal in $N$ we have $f_{i_{o}}(K)$ normal in $M_{i_{o}}$. By condition $(E)$ there exists a non-zero $M \in \mathcal{M}$ such that $\left[f_{i_{o}}(K), M\right]_{\mathscr{g}(\boldsymbol{G})} \neq \emptyset$. Since $K \rightarrow f_{i_{o}}(K)$ is a cokernel we have $[K, M]_{\mathscr{(}(\varepsilon)} \neq \emptyset$ and hence $\mathcal{N}$ also satisfies condition $(E)$. By Lemma 4.2 the $\mathscr{R}$-functor $\operatorname{Rad}_{\mathcal{M}}$ is complete.

\section{Special radical functors}

We introduce a new construction reminiscent of the upper radical for rings.

Definition 5.1. Let $\mathcal{M}$ be a non-empty class of objects of $\mathscr{C}$. Define $\mathscr{S}_{\mathcal{M}}=$ $\left\{R \in \mathscr{C} \mid[R, M]_{\mathscr{Q}(\mathscr{Q})}=\emptyset, \forall 0 \neq M \in \mathcal{M}\right\}$. Now for each object $C$ of $\mathscr{C}$ we form the union of all the normal subobjects of $C$ that are also objects of $\mathscr{S}_{\mu}$. We call this union the upper M-object of $C$ and denote it by $U \mathscr{S}_{\mathcal{M}}(C) .{ }^{1}$

It is immediate from the definitions that $U \mathscr{S}_{\mu}(C)$ is a normal subobject of $C$, for any $C \in \mathscr{C}$, and furthermore this construction is idempotent in the following sense:-

Proposition 5.2. Let $\mathcal{M}$ be a non-empty class of objects of $\mathscr{C}$. For each object $C$ in $\mathscr{C}, \mathcal{U} \mathscr{S}_{\mathcal{M}}(C)$ is a normal subobject of $C$ and $U \mathscr{S}_{\mathcal{M}}\left(U \mathscr{S}_{\mathcal{M}}(C)\right)=\mathcal{U} \mathscr{S}_{\mathcal{M}}(C)$.

Proof. Clearly for any $C \in \mathscr{C}, \mathcal{U} \mathscr{S}_{\mu}\left(\mathcal{U} \mathscr{S}_{\mu}(C)\right) \subseteq \mathscr{U} \mathscr{S}_{\mu}(C)$. Now suppose that $X$ is any normal subobject of $C$ that belongs to $\mathscr{S}_{\mathcal{M}}$, it lies in $\mathscr{U}_{\mathcal{\mu}}(C)$ and is also normal in $\mathcal{U} \mathscr{S}_{\mu}(C)$ and consequently belongs to $U \mathscr{S}_{\mu}\left(U \mathscr{S}_{\mu}(C)\right)$.

"Clearly 0 is in $\mathscr{S}_{\mu}$ and so $0 \subseteq U \mathscr{S}_{\mu}(C)$. 
In general, this construction may not give rise to a radical functor. However, in the category $\mathscr{R} i$ of rings, when $\mathcal{M}$ satisfies certain conditions we obtain the upper radical defined by $\mathcal{M}$.

If we start with a general class of objects $\mathcal{M}$ of $\mathscr{C}$, we can then describe two basic constructions, namely

$$
\operatorname{Rad}_{\mu}(C) \text { and } \mathscr{U} \mathscr{S}_{\mu}(C) \text { for any } C \in \mathscr{C} .
$$

In the general case there is no clear relationship between these two constructions but in the category of rings they are equal in certain circumstances, for example when the class $\mathcal{M}$ is a special class of rings.

In the general situation we adapt the definition of a special class of rings to the abstract categorical context and then prove a generalisation of the result in ring theory, namely that for a special class of objects the two constructions $\operatorname{Rad}_{\mu}(C)$ and $\mathcal{U} \mathscr{S}_{\mathcal{M}}(C)$ are equal for all $C \in \mathscr{C}$. An immediate corollary of this result yields the fact that $\operatorname{Rad}_{\mathcal{M}}$ is an idempotent $\mathscr{R}$-functor.

The procedure of transforming the concept of a special class of rings into a categorical setting requires some adaptation to make it suitable for future applications, including the theory of near-rings.

We have to introduce some terminology. As usual $\mathscr{C}$ will denote an $\boldsymbol{R}$-category with unions of normal subjects. Let $\mathscr{L}(\mathscr{C})$ be the category of short exact sequences in $\mathscr{C}$ and define $\mathscr{L}^{*}(\mathscr{C})$ to be the subcategory of $\mathscr{L}(\mathscr{C})$ formed as follows:- the objects of $\mathscr{L}^{*}(\mathscr{C})$ are triples of the form $A \rightarrow B \rightarrow C$ where $A \rightarrow B$ is a kernel of $\mathscr{C}$ and $B \rightarrow C$ is a cokernel of $\mathscr{C}$. If $A \rightarrow B \rightarrow C$ and $A^{\prime} \rightarrow B^{\prime} \rightarrow C^{\prime}$ are objects of $\mathscr{L}^{*}(\mathscr{C})$, the morphisms are commutative diagrams of the form

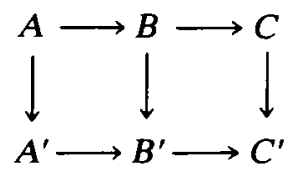

where $A \rightarrow A^{\prime}, B \rightarrow B^{\prime}, C \rightarrow C^{\prime}$ are all cokernels of $\mathscr{C}$.

Definition 5.3. Let $F: \mathscr{L}^{*}(\mathscr{C}) \rightarrow \mathscr{L}(\mathscr{C})$ be a covariant functor and suppose that $F(A \rightarrow B \rightarrow C)=A_{F} \rightarrow B_{F} \rightarrow C_{F}$ where $(A \rightarrow B \rightarrow C) \in \mathscr{L}^{*}(\mathscr{C})$ and $\left(A_{F} \rightarrow B_{F} \rightarrow C_{F}\right) \in$ $\mathscr{L}(\mathscr{C})$. We call $F$ a neutral functor if $B_{F}=B$ is always true.

Example 5.4. The following are neutral functors in the case $\mathscr{C}=\mathscr{R} i$, the category of rings.

(i) $F_{1}:(A \rightarrow K \rightarrow K / A) \rightarrow\left(A_{r} \rightarrow K \rightarrow K / A_{r}\right)$ where $A_{r}$ is the right annihilator of the ideal $A$ in the ring $K$

(ii) $F_{2}:(A \rightarrow K \rightarrow K / A) \rightarrow\left(A^{*} \rightarrow K \rightarrow K / A^{*}\right)$ where $A^{*}$ is the two-sided annihilator of the ideal $A$ in the ring $K$.

Definition 5.5. Let $F: \mathscr{L}^{*}(\mathscr{C}) \rightarrow \mathscr{L}(\mathscr{C})$ be a neutral functor and $\mathscr{X}$ a class of objects of $\mathscr{C}$. We call $\mathscr{Z}$ an $F$-special class if and only if:

(i) $(A \rightarrow B \rightarrow C) \in \mathscr{L}^{*}(\mathscr{C})$ with $B=A_{F}$ and $A \in \mathscr{L}$ implies $A=0$, 
(ii) $A \in \mathscr{Z}$ implies that all non-zero normal subobjects of $A$ belong to $\mathscr{X}$,

(iii) $(A \rightarrow B \rightarrow C) \in \mathscr{L}^{*}(\mathscr{C})$ with $A \in \mathscr{X}$ implies $C_{F} \in \mathscr{X}$,

(iv) $T$ is a normal subobject of $K, I$ is a normal subobject of $T$ and $T / I \in \mathscr{X}$ implies $I$ is a normal subobject of $K$,

(v) $\mathscr{Z}$ is closed under isomorphisms.

In some categories and with certain types of neutral functor the condition (iv) above is unnecessary since it can be deduced from the others. Condition (i) is related to the concept of a prime ring in the category $\mathscr{R} i$ with $F$ either of the functors in Example 5.4. Using this notion of an $F$-special class we can deduce an important result concerning the equality of the two main constructions that are under study. $\operatorname{Rad}_{\mathcal{M}}$ and $\mathscr{U} \mathscr{S}_{\mu}$. It is also possible to show that a certain type of primitive near-ring gives rise to an $F$-special class and this provides the application for the theory in the next section.

Theorem 5.6. Let $F: \mathscr{L}^{*}(\mathscr{C}) \rightarrow \mathscr{L}(\mathscr{C})$ be a neutral functor and $\mathscr{M}$ an $F$-special class of objects of $\mathscr{C}$. Then, for each $C \in \mathscr{C}$

$$
\operatorname{Rad}_{\mu} C=\mathscr{U} \mathscr{S}_{\mu}(C)
$$

Proof. Choose any $C \in \mathscr{C}$ and let $H(\mathcal{M}, C)$ be the set of the normal subobjects of $C$ belonging to $\mathscr{S}_{\mathcal{M}}$. Then $\mathscr{U}_{\mathcal{M}}(C)=\cup_{P \in H(\mathcal{M}, C)} P$. Now let $P \in H(\mathcal{M}, C)$, so that $[P, M]_{\&(\xi)}=\emptyset$ for all $0 \neq M \in M$. Then $\operatorname{Rad}_{\mathcal{M}} P$ is the empty intersection and so $\operatorname{Rad}_{\mathcal{M}} P=P$. Since $\mathcal{M}$ satisfies condition (ii) of Definition 5.5 it also satisfies condition $(E)$ of 4.1 and by Theorem $4.3, \operatorname{Rad}_{\mathcal{H}}$ is complete. Therefore $P$ is a subobject of $\operatorname{Rad}_{\mathcal{M}} C$. Clearly $\mathcal{U} \varphi_{\mathcal{M}}(C)$ is a subobject of $\operatorname{Rad}_{\mathcal{M}} C$.

Now let $A=\operatorname{Rad}_{\mu} C$ and suppose that $A$ is not $\mathcal{M}$-radical, i.e. there exists a non-zero $\alpha: A \rightarrow A / I$ with $I$ normal in $A$ and $A / I \in M$. Thus $A \notin H(\mathcal{M}, C)$. By (iv) of $5.5 I$ is normal in $C$ and so

$$
(A / I \rightarrow C / I \rightarrow(C / I) /(A / I)) \in \mathscr{L}^{*}(\mathscr{C}),
$$

and so by (iii) of 5.5, $((C / I) /(A / I))_{F} \in \mathcal{M}$. Now suppose that $((C / I) /(A / I))_{F}=(C / I) /(Q / I)$ for some normal subobject $Q$ of $C$ and where $Q / I=(A / I)_{F}$. Now $((C / I) /(Q / I)) \in M$ and so $C l Q \in \mathcal{M}$ as $\mathcal{M}$ is closed under isomorphisms. Since $C \rightarrow C / Q$ is a cokernel and $C / Q \in \mathcal{M}$ we have that $A$ is a subobject of $Q$. Thus

$$
(A / I \rightarrow Q / I \rightarrow(Q / I) /(A / I)) \in \mathscr{L}^{*}(\mathscr{C})
$$

with $A / I \in M, Q / I=(A / I)_{F}$ and so by (i) of $5.5, A / I=0$. Therefore $A$ must be $\mathcal{M}$-radical and so $A=\operatorname{Rad}_{\mathcal{M}} C$ is a subobject of $\mathcal{U S}_{\mathcal{M}}(C)$. This leads us to the final conclusion that

$$
\operatorname{Rad}_{\mu} C=\mathscr{U} \mathscr{S}_{\mathcal{M}}(C) \text { for any } C \in \mathscr{C}
$$

Corollary 5.7. Let $F: \mathscr{L}^{*}(\mathscr{C}) \rightarrow \mathscr{L}(\mathscr{C})$ be a neutral functor and $\mathcal{M}$ an $F$-special class of objects of $\mathscr{C}$. Then $\operatorname{Rad}_{\mu}: \mathscr{E}(\mathscr{C}) \rightarrow \mathscr{E}(\mathscr{C})$ is an idempotent and complete $\mathscr{R}$-functor.

Given an $F$-special class $\mathcal{M}$, for some neutral functor $F$ we can now talk about the 
$F$-special radical, $\operatorname{Rad}_{\mathcal{M}}(C)$ of an object $C$. We will also describe the functor $\operatorname{Rad}_{\mu}: \mathscr{E}(\mathscr{C}) \rightarrow \mathscr{C}(\mathscr{C})$ as an $F$-special $\mathscr{R}$-functor. Since there may be many types of neural functor associated with a category $\mathscr{C}$ it is possible that there are distinct types of $F$-special $\mathscr{R}$-functor, that is, an $\mathscr{R}$-functor may be $F$-special with respect to one $F$ but not with respect to another. However little seems to be known in this area. We turn now to a particular application of this theory.

\section{An application in the theory of near-rings}

The radical that has received the most study in the theory of near-rings ${ }^{11}$ is the radical $J_{2}$ (see, for example Betsch (1)). This radical is a generalisation of the Jacobson radical for rings and it inherits many of its properties. The radical $J_{2}$ may be defined as the $\mathscr{R}$-functor $\operatorname{Rad}_{\mathcal{M}}$ in the category of near-rings and near-ring homomorphisms where $\mathcal{M}$ is the class of 2-primitive near-rings. The situation in the theory of rings suggests that $\mathcal{M}$ may be $F$-special for some suitable neutral functor $F$ but this question is still open. We can however show that the class of 3-primitive near-rings is $F$-special for a particular $F$ and this is the content of this section.

Definitions 6.1. Let $N$ be a near-ring. An $N$-module (or $N$-group) $\Gamma$ is of type 3 if and only if:-

(i) $\Gamma \cdot N \neq(0)$

(ii) $\Gamma$ has no non-trivial $N$-subgroups

(iii) $\gamma \cdot n=\gamma^{\prime} \cdot n$ for all $n \in N \Rightarrow \gamma=\gamma^{\prime}$, where $\gamma, \gamma^{\prime} \in \Gamma$.

A near-ring $N$ is called 3-primitive if there exists a faithful $N$-module $\Gamma$ of type 3 .

We define now the class $\mathcal{M}_{3}$ of all 3-primitive near-rings. It is clear from the definition that a 3-primitive near-ring is 2-primitive but the converse it not true generally.

The radical $J_{3}$ is then defined to be the radical generated by the class $\mathcal{M}_{3}$. Thus, for any near-ring $N$.

$$
J_{3}(N)=\operatorname{Rad}_{\mu_{3}}(N)
$$

and so $J_{3}$ can be considered to be an $\mathscr{R}$-functor from $\mathscr{E}(\mathcal{N})$ to $\mathcal{N}$ where $\mathcal{N}$ is the category of near-rings and near-rings homomorphisms. We show that $J_{3}$ is in fact an $F$-special $\mathscr{R}$-functor for a suitable neutral functor $F: \dot{L}^{*}(\mathcal{N}) \rightarrow \mathscr{L}(\mathcal{N})$. First of all we define the neutral functor.

Let $F_{3}: \mathscr{L}^{*}(\mathcal{N}) \rightarrow \mathscr{L}(\mathcal{N})$ be defined by

$$
F_{3}(A \rightarrow K \rightarrow K / A)=\left(A_{r} \rightarrow K \rightarrow K / A_{r}\right)
$$

where $K$ is a near-ring, $A$ is an ideal of $K$ and $A_{r}$ is the right annihilator of $A$ in $K$. We claim:-

Theorem 6.2. $\quad J_{3}$ is an $F_{3}$-special $\mathscr{R}$-functor in the category $\mathcal{N}$.

Proof. The proof consists of verifying the conditions of Definition 5.5 in this particular case.

"All near-rings considered here satisfy the condition: $0 . n=0$ for all elements $n$ of the near-ring. 
(i) Let $(A \rightarrow K \rightarrow K / A) \in \mathscr{L}^{*}(\mathcal{N})$ with $A \in \mathscr{M}_{3}$ and $K=A_{r}$ If $\Gamma$ is an $A$-module of type 3 then $(\Gamma)_{r}=(0)$ but $A \subseteq K \subseteq A_{r}$ and so $\Gamma A=\gamma A A=0$ for some $0 \neq \gamma \in \Gamma$. Thus $A \subseteq(\Gamma)_{r}=(0)$ and so $A=(0)$.

(ii) Let $A \in \mathscr{M}_{3}$ and suppose that $I$ is a non-zero ideal of $A$. Let $\Gamma$ be an $A$-module of type 3 then $\Gamma$ is also an $I$-module. Note that $\Gamma I=0 \Rightarrow I \subseteq(\Gamma)_{r}$ but $(\Gamma)_{r}=(0)$ and so $I$ must then be zero. Furthermore, let $\Delta$ be an $I$-subgroup of $\Gamma$ and suppose that there exists $\delta \in \Delta$ with $\delta \neq 0$. Then $\delta I$ is an $A$-subgroup of $\Gamma$ and $\delta I=0$ or $\Gamma$. If $\delta I=\Gamma$ then $\Delta=\Gamma$, so we assume that $\delta I=0$; then $\delta A I=0$ and so $\Gamma I=0$ since $\delta A=\Gamma$ when $\delta \neq 0$. This contradiction establishes the fact that $\Gamma$ contains no non-trivial $I$-subgroups. Finally we suppose that $\gamma^{\prime}, \gamma^{\prime \prime} \in \Gamma$ exist such that $\gamma^{\prime} i=\gamma^{\prime \prime} i$ for all $i \in I$. There exists $e \in I$ such that $\gamma^{\prime}=\gamma^{\prime} e$, otherwise both $\gamma^{\prime}$ and $\gamma^{\prime \prime}$ are zero. The right annihilators in $N$ of $\gamma^{\prime}$ and $\gamma^{\prime \prime}$ are equal. Now $\gamma^{\prime} \cdot(n-e n)=0$ for all $n \in N$ and so $\gamma^{\prime \prime} \cdot(n-e n)=0$, thus $\gamma^{\prime \prime} n=\gamma^{\prime \prime} e n$ for all $n \in N$ and so $\gamma^{\prime \prime}=\gamma^{\prime \prime} e$. For any $n^{\prime} \in N$, $\gamma^{\prime} n^{\prime}-\gamma^{\prime \prime} \cdot n^{\prime}=\gamma^{\prime} \cdot e n^{\prime}-\gamma^{\prime \prime} \cdot e n^{\prime}=0$ since $e n^{\prime} \in I$ and so $\gamma^{\prime}=\gamma^{\prime \prime}$. Therefore $\Gamma$ is an $I$-module of type 3. Hence $I$ is 3-primitive i.e. $I \in \mathcal{M}_{3}$.

(iii) Let $(A \rightarrow K \rightarrow K / A) \in \mathscr{L}^{*}(\mathcal{N})$ with $A \in \mathscr{M}_{3}$ and consider $K / A_{r}$. We must show that $K / A_{r} \in \mathcal{M}_{3}$. First we define a $K$-module structure for an arbitrary faithful $A$-module $\Gamma$ of type 3. Given $\gamma \in \Gamma$ with $\gamma \neq 0$, then $\gamma A=\Gamma$ and so given any $\gamma^{\prime} \in \Gamma$ there exists $a^{\prime} \in A$ with $\gamma^{\prime}=\gamma \cdot a^{\prime}$. Now, for any $k \in K$ we define $\gamma^{\prime} \cdot k=\gamma \cdot\left(a^{\prime} k\right)$. This operation is well-defined, for suppose that $\gamma^{\prime}=\gamma \cdot a^{\prime \prime}\left(a^{\prime \prime} \in A\right)$, then, for any $k \in K$ and any $a \in A, k \cdot a \in A$ and $\left(\gamma \cdot a^{\prime}\right)(k a)=\left(\gamma \cdot a^{\prime \prime}\right)(k a)$. Therefore $\gamma\left(a^{\prime} k\right) a=$ $\gamma\left(a^{\prime \prime} k\right) a$ which holds for all $a \in A$ and implies $\gamma\left(a^{\prime} k\right)=\gamma\left(a^{\prime \prime} k\right)$. It is clear that $\Gamma$ is a $K$-module. Now we consider $K / A_{r}$ and note that $A \cap A_{r}=(0)$, since $\Gamma$ is faithful as an $A$-module. Furthermore for $x \in A_{n} \bar{\gamma}^{\prime} \cdot x=\gamma\left(\bar{a}^{\prime} x\right)=0$, where $\bar{\gamma}^{\prime}=\gamma \bar{a}^{\prime}$ is an element of $\Gamma$. Define $\bar{\gamma}^{\prime} \cdot\left(k+A_{r}\right)=\bar{\gamma}^{\prime} \cdot k$ for any $k \in K, \bar{\gamma}^{\prime} \in \Gamma$ and we see that $\Gamma$ is a $K / A_{r^{-}}$ module. Furthermore $\Gamma \cdot\left(K / A_{r}\right) \neq 0$ and $\Gamma$ contains no non-trivial $\left(K / A_{r}\right)$-subgroups. Finally if $\gamma_{1}^{\prime} \cdot\left(k+A_{r}\right)=\gamma_{1}^{\prime \prime}\left(k+A_{r}\right)$ for all $k+A_{r} \in K / A_{r}$ then $\gamma_{1}^{\prime} \cdot a=\gamma_{1}^{\prime \prime} \cdot a$ for all $a \in A$ and so $\gamma_{1}^{\prime}=\gamma_{1}^{\prime \prime}$. Therefore $\Gamma$ is a $K / A_{r}$-module of type 3 . Now if $x \in K$ with $\Gamma x=0$, then $\bar{\gamma} A x=0$ for any $\bar{\gamma} \in \Gamma$. Therefore $\Gamma A x=0$ and so $A x=0$ i.e. $x \in A_{r}$ Thus the annihilator of $\Gamma$ in $K / A_{r}$ is zero. Consequently therefore $K / A_{r}$ is 3-primitive and must belong to $\mathcal{M}_{3}$.

(iv) Suppose that $T$ is an ideal of $K, I$ is an ideal of $T$ and $T / I$ is 3-primitive. We must show that $I$ is an ideal of $K$. Let $\Gamma$ be a faithful $T / I$-module of type 3 . Then $\Gamma$ is, in a natural way, a $T$-module of type 3 and the annihilator of $\Gamma$ in $T,(I)_{r}$ is $I$. As before $\Gamma$ is also a $K$-module and we consider the annihilator of $\Gamma$ in $K$, denoted by $(\Gamma)_{r}^{K}$. This is an ideal of $K$ and, furthermore $I=T \cap(\Gamma)_{r}^{K}$. Since $I$ is the intersection of two ideals of $K$ it must also be an ideal of $K$.

(v) Finally it is straightforward to verify that if $A \in \mathcal{M}_{3}$ and $B$ is isomorphic to $A$ then $B \in \mathscr{M}_{3}$. This completes the proof. Therefore $J_{3}$ is $F_{3}$-special. Consequently for any near-ring $N$,

$$
J_{3}(N)=\operatorname{Rad}_{\mathcal{M}_{3}} N=U \mathscr{S}_{\mathcal{H}_{3}}(N)
$$

This completes the generalisation of the result in ring theory that states that the Jacobson radical of a ring is a special radical. Further, immediate deductions are that the $J_{3}$ radical for near-rings is complete and idempotent. 


\section{REFERENCES}

(1) G. BETSCH, Struktursatze für Fastringe, Doctoral Dissertation Eberhard-Karls. Universität zu Tübingen (1963).

(2) F. CARREAU, Sous-categories réflexives et la théorie générale des radicaux, Fund. Math. 71 (1971), 223-242.

(3) S. E. Dickson, A torsion theory for abelian categories, Trans. Amer. Math. Soc. 121 (1966), 223-235.

(4) N. J. DivinSKY, Rings and radicals, (George Allen and Unwin Ltd, 1965).

(5) J. M. MaRAnda, Injective structures, Trans. Amer. Math. Soc. 110 (1964), 98-135.

(6) B. MrtchelL, Theory of categories (Academic Press, 1965).

(7) E. G. SHUL'GEIFER, Functor characterisation of strict radicals in categories, Sib. Matem. Zh. 7, No. 6, (1966), 1412-1421.

(8) R. Wiegandt, Radical and semi-simplicity in categories, Acta. Math. Acad. Sc. Hung. 19 (1968), 345-364.

THE QUEEN'S UNIVERSITY OF BELFAST BELFAST BT7 1NN
TEMPLE MOOR HIGH SCHOOL FIELD END GROVE

SElby RoAd

LEEDS LS15 OPT 